\title{
Spectral analysis of fundus autofluorescence pattern as a tool to detect early stages of degeneration in the retina and retinal pigment epithelium
}

\author{
Tatiana B. Feldman $\mathbb{1}^{1,2} \cdot$ Marina A. Yakovleva ${ }^{2} \cdot$ Andrey V. Larichev $^{3,4} \cdot$ Patimat M. Arbukhanova $^{5} \cdot$ \\ Alexandra Sh. Radchenko ${ }^{2}$ - Sergey A. Borzenok ${ }^{5}$. Vladimir A. Kuzmin ${ }^{2} \cdot$ Mikhail A. Ostrovsky $^{1,2}$
}

Received: 29 October 2017 / Revised: 27 February 2018 / Accepted: 30 March 2018 / Published online: 22 May 2018

(c) The Royal College of Ophthalmologists 2018

\begin{abstract}
Purpose The aim of this work is the determination of quantitative diagnostic criteria based on the spectral characteristics of fundus autofluorescence to detect early stages of degeneration in the retina and retinal pigment epithelium (RPE).

Methods RPE cell suspension samples were obtained from the cadaver eyes with and without signs of age-related macular degeneration (AMD). Fluorescence analysis at an excitation wavelength of $488 \mathrm{~nm}$ was performed. The fluorescence lifetimes of lipofuscin-granule fluorophores were measured by counting time-correlated photon method.

Results Comparative analysis of fluorescence spectra of RPE cell suspensions from the cadaver eyes with and without signs of AMD showed a significant difference in fluorescence intensity at $530-580 \mathrm{~nm}$ in response to fluorescence excitation at $488 \mathrm{~nm}$. It was notably higher in eyes with visual pathology than in normal eyes regardless of the age of the eye donor. Measurements of fluorescence lifetimes of lipofuscin fluorophores showed that the contribution of photooxidation and photodegradation products of bisretinoids to the total fluorescence at $530-580 \mathrm{~nm}$ of RPE cell suspensions was greater in eyes with visual pathology than in normal eyes.

Conclusion Because photooxidation and photodegradation products of bisretinoids are markers of photodestructive processes, which can cause RPE cell death and initiate degenerative processes in the retina, quantitative determination of increases in these bisretinoid products in lipofuscin granules may be used to establish quantitative diagnostic criteria for degenerative processes in the retina and RPE.
\end{abstract}

Tatiana B. Feldman

feldmantb@mail.ru

1 Department of Molecular Physiology, Biological Faculty, Lomonosov Moscow State University, Leninskie Gory 1, Moscow, Russia 119991

2 Emanuel Institute of Biochemical Physics, Russian Academy of Sciences, Kosygin st.4, Moscow, Russia 119334

3 The Department of Medical Physics, Faculty of Physics, Lomonosov Moscow State University, Leninskie Gory 1, Moscow, Russia 119991

4 Federal Scientific Research Center Crystallography and Photonics, Institute on Laser and Information Technologies of Russian Academy of Sciences, Svyatoozerskaya st. 1, Shatura, Moscow Region, Russia 140700

5 Sv. Fyodorov Eye Microsurgery Complex, Beskudnikovsky bld. 59a, Moscow, Russia 127486

\section{Introduction}

Noninvasive fundus autofluorescence (FAF) imaging is one of the most promising procedures for diagnosing various visual pathologies [1]. The main source of FAF is lipofuscin granules (LGs), which accumulate in retinal pigment epithelium (RPE) cells of the human eye during aging, particularly in patients with hereditary diseases $[2,3]$, including progressive age-related macular degeneration (AMD) [4, 5]. LGs are formed by the incomplete lysosomal degradation of outer-segment photoreceptor cell debris resulting from the phagocytosis of RPE cells [6]. In addition, other components of cells, including flavin adenine dinucleotide (FAD), nicotinamide adenine dinucleotide phosphate (NADPH), and the carotenoids zeaxanthin and lutein, have weak fluorescence in the same spectral area as LGs [7-9]. In addition, spectral autofluorescence characteristics of drusen have been observed in donor eyes with AMD [10]. 
LGs are heterogeneous, composed of mixtures of proteins and lipids, including more than 21 different fluorescent compounds [11-15]. Bisretinoids, and their photooxidation and photodegradation products, represent the main sources of LG fluorescence [16, 17]. Among the compounds investigated in LGs are the bisretinoid $\mathrm{N}$-retinylidene- $\mathrm{N}$ retinylethanolamine (A2E) [18-20], photooxidation and photodegradation products of bisretinoids [17, 21-25], and a series of all-trans retinal conjugates in LGs [26-28].

FAF images are usually obtained with a confocal scanning laser ophthalmoscope (cSLO) [29, 30], but may also be obtained with a fundus camera, with the two methods generally showing a high degree of correlation. Differences between the two devices have also been observed. For example, multiple image capture and confocal optics yielded higher image contrast with a cSLO, despite acquisition and exposure times being longer [31].

FAF is excited by a wavelength of $488 \mathrm{~nm}$, yielding monochromatic images in the long-wavelength region, starting at $500 \mathrm{~nm}$, resulting from the fluorescence of bisretinoids and their photooxidation and photodegradation products.

FAF can detect early phenotypic changes in RPE, occurring prior to the progression of disease. Analysis of FAF patterns can provide detailed qualitative information that allows the detection of areas of pathology, thereby differentiating among different types of ocular disease. At present, however, it is not possible to quantify the detected changes. The degree of disease progression must be assessed subjectively by an expert, who compares patterns with those of normal eyes.

Since its introduction into ophthalmic practice, efforts have been made to improve the FAF technique to obtain additional information from the total fluorescence patterns. For example, the spectral characteristics of FAF may be used to determine the occurrence of degenerative processes in the retina and RPE [32-35], with one study proposing a method and apparatus for determining fluorophores on objects, especially on the living ocular fundus [33]. Based on the fluorescence behavior over time at each site, as determined by timecorrelated single-photon counting, the fluorescence time constants can be calculated. Tissue function and metabolism can be determined noninvasively by steady-state fluorescence anisotropy [35]. Systems and methods have been described for optical detection of lipofuscin concentration in a subject's eye [34]. Moreover, retinal diseases may be distinguished by comparing autofluorescent emissions at different wavelengths (e.g., $410-530 \mathrm{~nm}$, corresponding to the fluorophores of Bruch's membrane and drusen, and $505-700 \mathrm{~nm}$, corresponding mainly to the fluorophores of LGs in RPE) [32]. In addition, an increase in blue-green autofluorescence of Bruch's membrane relative to the yellow-orange autofluorescence of RPE-associated lipofuscin is associated with AMD [36]. Fluorescence lifetime imaging microscopy
(FLIM) at certain wavelengths is an extremely promising approach [37-40], with patterns of FLIM in vivo differing in normal eyes and those with AMD [41]. In addition, changes from normal fluorescence parameters were observed in patients with diabetes [42], glaucoma [43], and Alzheimer's disease [44]. All of these findings were confirmed by experimental research, which showed that the quantitative and qualitative composition of LG fluorophores changes during pathologic development $[17,25]$.

One of the main objectives of the FAF and FLIM methods is to determine the differences in fluorescent characteristics of $L G$ fluorophores between individuals with ocular pathology and those with normal eyes. We recently showed [17] that the relative contents of photooxidation and photodegradation products of bisretinoids in LGs change both with age and with the development of ocular pathology. However, a shortwave shift in maximum fluorescence is observed only in pathologic conditions. This study continues our investigation of the fluorescence properties of LGs in the RPE isolated from normal human cadaver eyes and those with AMD.

\section{Methods}

Experiments on tissue isolated from human cadaver eyes complied with officially accepted procedures, in particular Russian Federation law N 4180-I dated 12.22.1992, "On human organs or tissue transplantation" (with modifications and additions dated 06.20.2000, 10.16.2006, 02.09.2007, 11.29.2007, and 05.23.2016) [45]. Human cadaver eyes were obtained from the Eye Tissue Bank of the S.N. Fyodorov Eye Microsurgery Research and Technology Complex, Ministry of Health of the Russian Federation (Moscow, Russia). The Eye Tissue Bank granted permission for the use of cadaver eyes for research, in accordance with local ethics requirements, as described [17].

Human cadaver eyes were obtained within $10 \mathrm{~h}$ of donor death, following corneal removal for transplantation. Each cadaver eye was subject to postmortem inspection by an ophthalmologist and ophthalmic pathologist. After removal of the lens, vitreous body, and retina, a detailed description of the fundus was carried out and photographed. The age and sex of each eye donor were recorded, as well.

Human cadaver eyes with no signs of pathology from 11 donors aged $27(n=2), 32(n=1), 39(n=2), 45(n=2)$, $46(n=2), 58(n=1), 60(n=1), 65(n=2), 67(n=2), 68$ $(n=2)$, and $74(n=2)$ years were obtained. The RPE in the fundus of the eye without signs of pathology was uniform, without focal abnormalities, with the RPE cells in the foveolar area having more intense pigmentation. Figure 1 (norm) shows the typical image of the fundus for all cadaver eyes with no signs of pathology. 


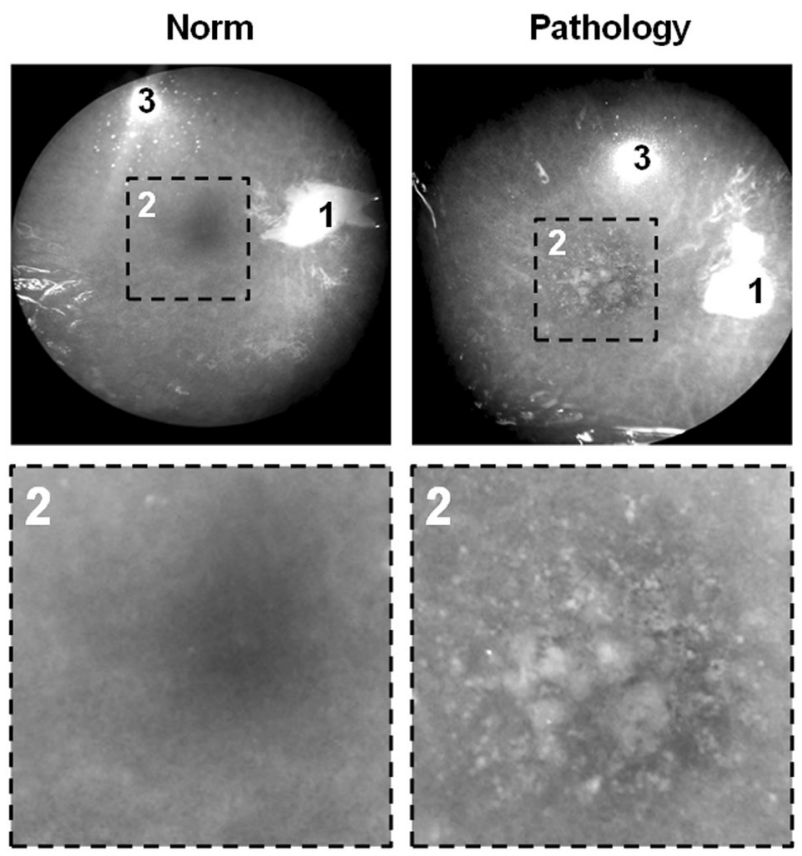

Fig. 1 Photographs of human cadaver eye fundus after removal of the retina from a normal eye (67-year-old donor) and an eye with signs of AMD (67-year-old donor). 1-disk of the optic nerve; 2-macular area; and 3-reflection from the microscope illumination

Human cadaver eyes with signs of pathology (AMD) from eight donors aged $59(n=1), 67(n=2), 70(n=1)$, $74(n=1), 75(n=2), 79(n=1), 87(n=2)$, and $88(n=2)$ years were obtained. The RPE in the fundus of the eye with AMD was uneven, with a redistribution of the pigment in the paramacular area. Such eye showed the presence of multiple drusen-like deposits, which were prominent and yellowish in color, and had irregular borders, surrounded by an area of hyperpigmentation. The presence of drusen-like deposits between the RPE and Bruch's membrane was confirmed by histological analysis (data not shown). Figure 1 (pathology) shows the typical image of the fundus for all cadaver eyes with signs of pathology.

It should be noted that the selection of cadaver's eyes for both the norm and pathology was carried out very carefully. Human cadaver eyes with questionable signs of norm or pathology were not used for comparative analysis.

All stages of sample preparation were carried out under subdued lighting.

\section{Preparation of suspended RPE cells from cadaver eyes}

The sclera of each cadaver eye was incised with scissors, and the iris, lens, vitreous body, and retina were removed. The eyecup with the naked RPE layer was placed in $1 \mathrm{ml}$ of $0.1 \mathrm{~mol} / \mathrm{l}$ phosphate buffer ( $\mathrm{pH} 7.2-7.4)$. Desquamation of the RPE surface from the macula zone was performed using a microsurgical spatula, with each cell suspension aspirated into a disposable pipette and transferred to a cooled flask.

\section{Preparation of chloroform extracts of cadaver eye RPE}

The soluble fraction was extracted from the samples with chloroform:methanol using the Folch method [46]. Briefly, a twofold excess of chloroform:methanol $(2: 1, \mathrm{vol} / \mathrm{vol})$ was added to each RPE suspension, agitated with an electrical stirrer for $2 \mathrm{~min}$, and incubated for $10 \mathrm{~min}$ at $4{ }^{\circ} \mathrm{C}$. The mixture was centrifuged at $680 \times g$ for $10 \mathrm{~min}$ at $4^{\circ} \mathrm{C}$. The bottom chloroform phase was aspirated into a syringe, transferred to a flask, and evaporated using a vacuum pump (Vacuubrand $\mathrm{MZ}$ 2CNT $+\mathrm{AK}+\mathrm{M}+\mathrm{D}$, Germany). For further chromatographic analysis, each dried sample was resuspended in $200 \mu$ l of methanol.

\section{High-performance liquid chromatography (HPLC)}

The compound was passed through a Knauer chromatograph system (Germany) equipped with a Kromasil-100-5C18 $(4 \times 250 \mathrm{~mm}$; sorbent size, $5 \mu \mathrm{m})$ column. Highperformance liquid chromatography (HPLC) analysis was performed using a reverse-phase gradient from $80 \%$ acetonitrile $/ 20 \%$ water $(\mathrm{vol} / \mathrm{vol})(+0.05 \% \mathrm{TFA})$ to $100 \%$ acetonitrile for $20 \mathrm{~min}$ at a flow rate of $1.0 \mathrm{ml} / \mathrm{min}$. Absorbance (K-2501 detector, Knauer) was measured at $430 \mathrm{~nm}$.

\section{A2E synthesis}

A2E was prepared from all-trans retinal and ethanolamine in acetic acid and ethanol, as described [47]. The purity of $\mathrm{A} 2 \mathrm{E}$ and iso-A2E was monitored by HPLC.

\section{Spectroscopy}

Fluorescence data were obtained using a RF-5301 PC fluorometer (Shimadzu, Japan) equipped with a R955 photomultiplier tube detector (Hamamatsu, Japan). Data were compiled using RFPC software version 2.0 (Shimadzu, Japan). Following excitation at $488 \mathrm{~nm}$, emission spectra were recorded at sampling intervals of $1 \mathrm{~nm}$. These fluorescence spectra were corrected for excitation intensity by the spectral response (quantum efficiency) of the R955 photomultiplier tube detector. All fluorescence spectra were normalized to a wavelength of $592 \mathrm{~nm}$.

\section{Method of counting time-correlated single photons}

The fluorescence lifetimes of LG fluorophores in the RPE cell suspension were measured by counting time-correlated photons with a FluoTime 300 spectrometer (PicoQuant, 
$\mathrm{GmbH}$, Germany). Fluorescence was excited using a LDHPC-450 diode laser ( $485 \mathrm{~nm}$; pulse duration, $30 \mathrm{ps}$ ), and fluorescence signals were recorded at wavelengths of 540 and $620 \mathrm{~nm}$. The fluorescence decay kinetic curves were processed in a time range during which light from the excitation pulse did not contribute to the detected fluorescence. Characteristic times and the contribution of individual components to the detected fluorescence were calculated using a three-exponential model, without accounting for the effect of the excitation pulse, using the following formula:

$\Phi(t)=\sum_{i} A_{i} \exp \left(-t / \tau_{i}\right)$

where $i$ is the number of components, $A$ is the amplitude that determines the contribution of each component, and $\tau$ is the duration of the components. The $\tau_{1}$ values are considered approximate, because the time characteristics of this short-lived component are comparable to the duration of the excitation pulse. Therefore, this parameter was not included in this study. Only the long-lived components $\tau_{2}$ and $\tau_{3}$ were included.

\section{Results}

\section{Comparative fluorescence characteristics of LG fluorophores in RPE cells from cadaver eyes with and without signs of AMD}

Fluorescence analysis at an excitation wavelength of 488 $\mathrm{nm}$, identical to that used in cSLO, was performed on separate RPE cell suspension samples from each cadaver eye. Figure $2 \mathrm{a}$ shows the example of the fluorescence spectra of RPE cell suspensions obtained from the eyes with no signs of pathology and with AMD. In both cases, the fluorescence spectra have two characteristic bands, with maxima at 556 and $592 \mathrm{~nm}$. The fluorescence spectrum of RPE cell suspension from the normal eye had a more pronounced maximum at $592 \mathrm{~nm}$, with the maximum at $556 \mathrm{~nm}$ representing an arm. By contrast, the spectrum of RPE cell suspension from the donor eye with signs of AMD had a more pronounced maximum at $556 \mathrm{~nm}$, indicating a greater accumulation of photooxidation and photodegradation products of bisretinoids in LGs from AMD than from normal eyes [17]. To confirm this finding, suspensions of these RPE cells were extracted with chloroform and analyzed by HPLC (Fig. 2b). The sample obtained from the eye with signs of AMD had a more diversified set of peaks, corresponding to the photooxidation and photodegradation products of $\mathrm{A} 2 \mathrm{E}$, at the initial retention time. Thus, fluorescence analysis of the RPE cell suspensions confirmed results showing
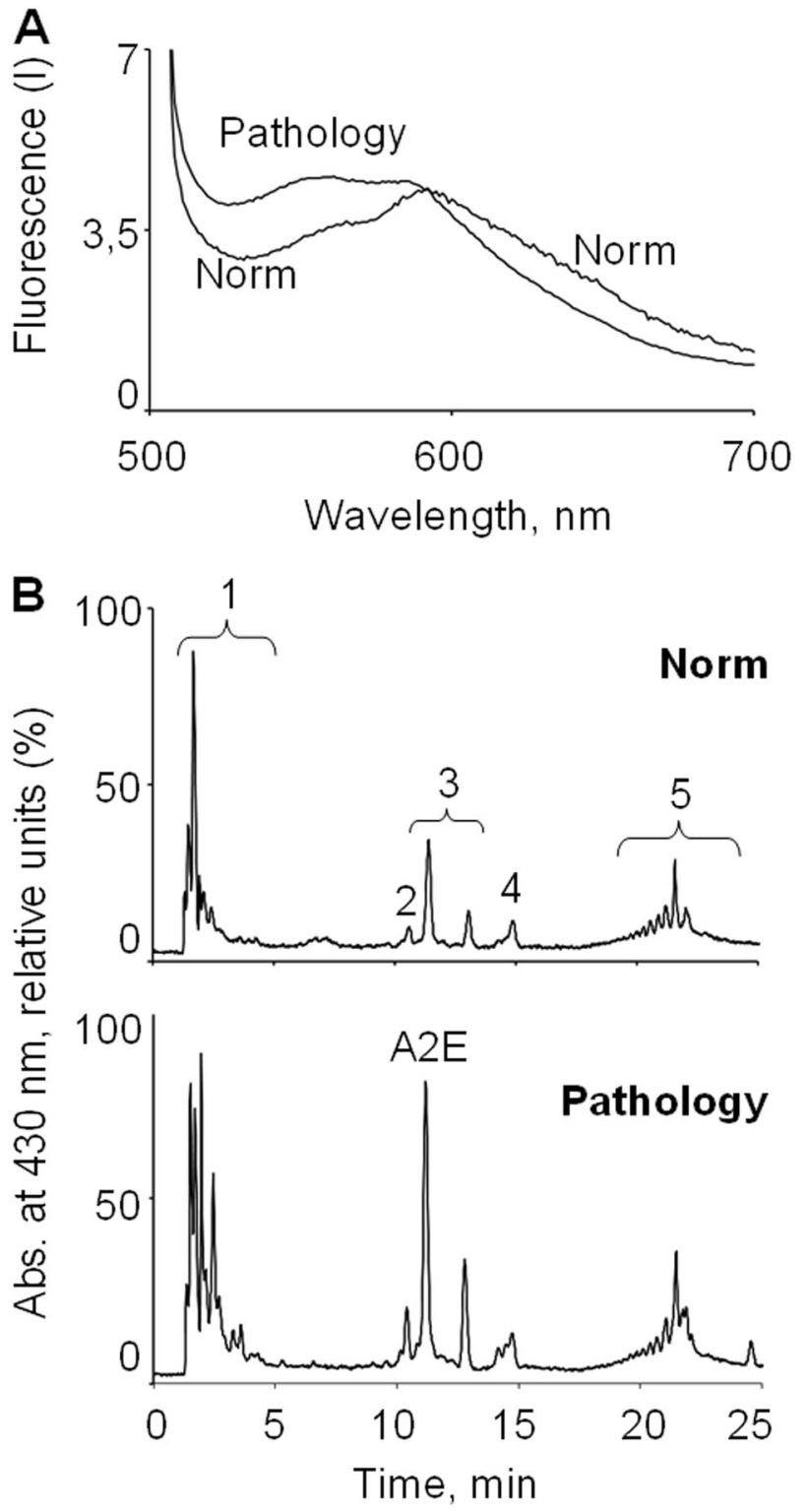

Fig. 2 Comparative analysis of spectral characteristics and composition of LG fluorophores in the RPE cells of human cadaver eyes with no signs of pathology (Norm) from a 67-year-old donor, and with signs of AMD (Pathology) from a 67-year-old donor. Photographs of eye fundus from these donors can be seen in Fig. 1. a Fluorescence emission spectra of these RPE cell suspensions. The excitation wavelength was $488 \mathrm{~nm}$, with emission spectra normalized at $592 \mathrm{~nm}$. b HPLC analysis of chloroform extracts of RPE cell suspensions, with absorption monitored at $430 \mathrm{~nm}$. Peaks 1 and 2 represent the photooxidation and photodegradation products of $\mathrm{A} 2 \mathrm{E}$ [17], peak 3 represents $\mathrm{A} 2 \mathrm{E}$ and iso-A2E [17], and peaks 4 and 5 correspond to other previously identified products $[26,27]$

differences between the fluorescence spectra of samples from normal eyes and eyes with AMD.

In contrast to our previous study, which analyzed only two eyes with AMD [17], this study analyzed 12 eyes with AMD from eight donors, enabling a comparative statistical 

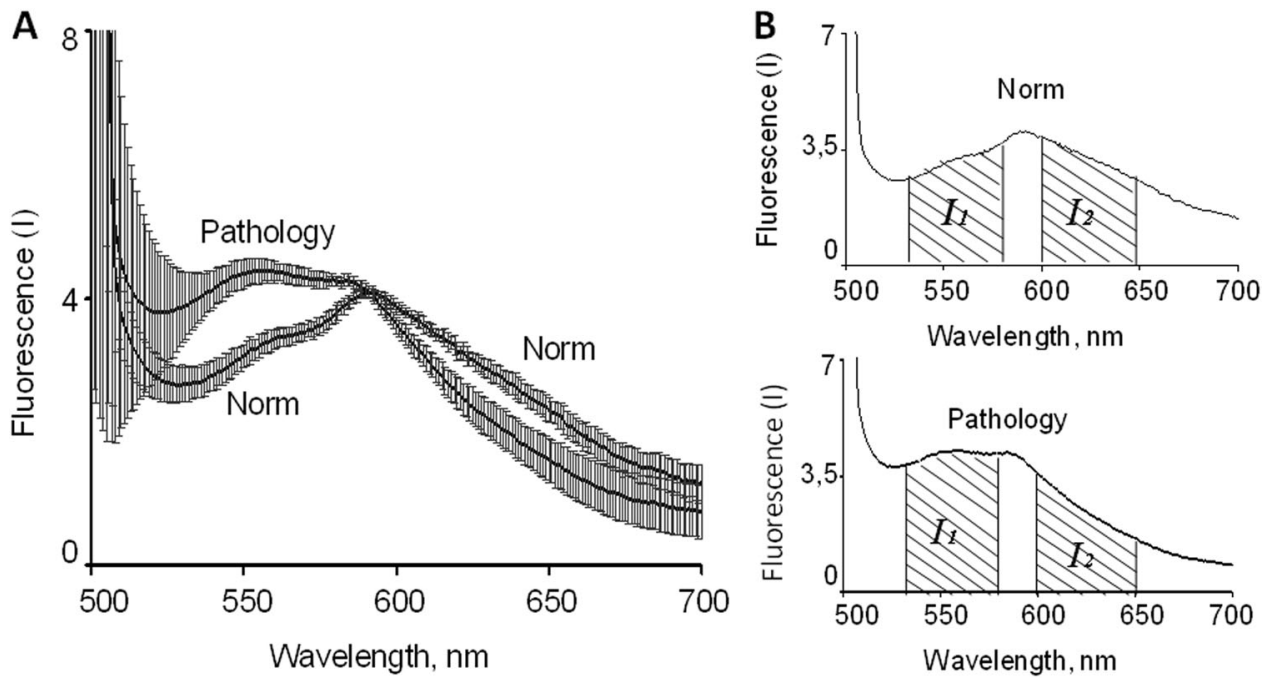

Fig. 3 a Comparative statistical analysis of spectral characteristics of RPE cell suspensions from cadaver eyes without (Norm) and with (Pathology) signs of AMD. Fluorescence spectra were averaged for 19 normal eyes, from 11 donors aged $27(n=2), 32(n=1), 39(n=2)$, $45(n=2), 46(n=2), 58(n=1), 60(n=1), 65(n=2), 67(n=2)$, $68(n=2)$, and $74(n=2)$ years, and for 12 AMD eyes, from eight donors aged $59(n=1), 67(n=2), 70(n=1), 74(n=1), 75(n=2)$,

analysis of the fluorescent properties of RPE cell suspensions of normal and AMD eyes (Fig. 3a).

Spectra were obtained by averaging the individual fluorescence spectra of all samples, including the standard deviation at each point of the spectrum. The average fluorescence spectrum for the normal samples showed a pronounced maximum in the region of $592 \mathrm{~nm}$ and a shoulder at $556 \mathrm{~nm}$, whereas the average fluorescence spectrum of AMD samples showed greater band intensity at $556 \mathrm{~nm}$ than that of the normal samples. This difference was again due to the higher content of bisretinoid photooxidation and photodegradation products in LGs from eyes with AMD. Normalization of all spectra relative to the fluorescence intensity at $592 \mathrm{~nm}$ showed that the fluorescence intensity in the AMD samples was markedly increased at $530-580 \mathrm{~nm}$ but reduced at $600-650 \mathrm{~nm}$.

Our previous study [17], using an excitation wavelength of $430 \mathrm{~nm}$, showed a difference in the fluorescence spectra of normal and AMD samples. However, that difference manifested as a noticeable shift in the maximum of the fluorescence spectrum at short wavelengths. Moreover, the previous study assessed samples from only two eyes with AMD, preventing a determination of the average spectral characteristics of normal and AMD eyes. By contrast, this study, which used an excitation wavelength of $488 \mathrm{~nm}$, found that the shapes of the spectra, after normalization at $592 \mathrm{~nm}$, were almost identical for normal eyes and for eyes with AMD. That is, spectral characteristics were determined only by the presence or absence of pathological changes in $79(n=1), 87(n=2)$, and $88(n=2)$ years. The excitation wavelength was $488 \mathrm{~nm}$, with emission spectra normalized at $592 \mathrm{~nm}$. b Fluorescence emission spectra of RPE cell suspensions obtained from individual cadaver eyes of a normal donor aged 58 years and a donor with signs of AMD aged 59 years. The excitation wavelength was $488 \mathrm{~nm}$. The integral intensities at spectral ranges $I_{1}(530-580 \mathrm{~nm})$ and $I_{2}$ $(600-650 \mathrm{~nm})$ were determined after normalizing the spectra at $592 \mathrm{~nm}$

the RPE, while being almost independent of donor age. Thus, these fluorescence characteristics have reproducible features that could be used diagnostically to evaluate ocular pathology. The ratio of fluorescence intensity in the shortwave part of the spectrum $(530-580 \mathrm{~nm})$ to the fluorescence intensity in the long-wavelength part $(600-650 \mathrm{~nm})$ may therefore be considered a diagnostic criterion.

These results suggest a method of calculating this ratio for diagnostic purposes. First, the fluorescence spectrum should be registered, using an excitation wavelength of 488 $\mathrm{nm}$. Second, the integral intensities of fluorescence $I_{1}$ and $I_{2}$ in the spectral ranges $530-580 \mathrm{~nm}$ and $600-650 \mathrm{~nm}$, respectively, should be calculated (Fig. 3b). These integrated intensities can be calculated using the following equations:

$$
\begin{aligned}
& I_{1}=\sum_{x, y \in \Omega} I_{1}(x, y) \\
& I_{2}=\sum_{x, y \in \Omega} I_{2}(x, y)
\end{aligned}
$$

where $x$ and $y$ are coordinates of a point in the fundus (or in the image of the fundus) within a given region $\Omega$. A quantitative parameter characterizing the difference in spectral characteristics of FAF in normal eyes and eyes with AMD, for use as a diagnostic criterion, can be calculated as their ratio:

$$
K=I_{1} / I_{2}
$$


Based on our data (Fig. 3), the calculated values for normal $\left(K_{\mathrm{N}}\right)$ and $\mathrm{AMD}\left(K_{\mathrm{P}}\right)$ eyes were $1.04 \pm 0.11$ and 1.73 \pm 0.16 , respectively. The separation between normal and AMD eyes allows quantitative diagnosis of the occurrence of degenerative processes. A value greater than 1.15, in the absence of an autofluorescence pattern indicative of pathology, may suggest that LGs in the RPE cells contain higher-than-normal amounts of bisretinoid photooxidation and photodegradation products. These products are regarded as markers of photodestructive processes, which can cause cell death and initiate degenerative processes in the retina. Thus, use of this criterion may enable the early detection of a pathological process, when FAF imaging results do not differ from those of normal eyes. Because these spectral analysis techniques are highly sensitive, a $K$ value higher than 1.15 may indicate a very early phase of the disease.

\section{Comparative analysis of fluorescence decay kinetics of LG fluorophores in RPE cell suspensions from cadaver eyes with and without signs of AMD}

In addition to the spectral examination, the difference between normal and AMD eyes can be evaluated by determining the kinetics of fluorescence decay [38]. In this study, fluorescence decay kinetics were determined using a method of counting time-correlated single photons. The contribution of different LG fluorophores (namely, bisretinoids and their photooxidation and photodegradation products) to the total fluorescence of RPE cell suspensions was compared between two cadaver eyes from a 75-year-old donor with signs of AMD and two cadaver eyes from a 74year-old donor without signs of pathology. The fluorescence spectra of all four RPE suspensions were recorded (Fig. 4a).

The spectral characteristics of the fluorescence spectra from normal and AMD eyes were similar to those shown previously (Fig. 2). That is, the fluorescence intensity of the band at $556 \mathrm{~nm}$ was greater in AMD than in normal eyes, indicating a greater accumulation of bisretinoid photooxidation and photodegradation products in LGs from AMD eyes. Using the time-correlated single-photon counting procedure, we measured the fluorescence lifetime of LG fluorophores in all RPE cell suspensions. Fluorescence signals were recorded at 540 and $620 \mathrm{~nm}$, the wavelengths showing the greatest difference in fluorescence intensity between normal and AMD eyes (Fig. 4b).

LGs contain more than 21 fluorophores, most of which are bisretinoids and their photooxidation and photodegradation products $[17,25]$. Because only the fluorophore A2E has been studied comprehensively [18-20], it was impossible to perform a detailed analysis of kinetic curves in a multicomponent system. In the present study, we
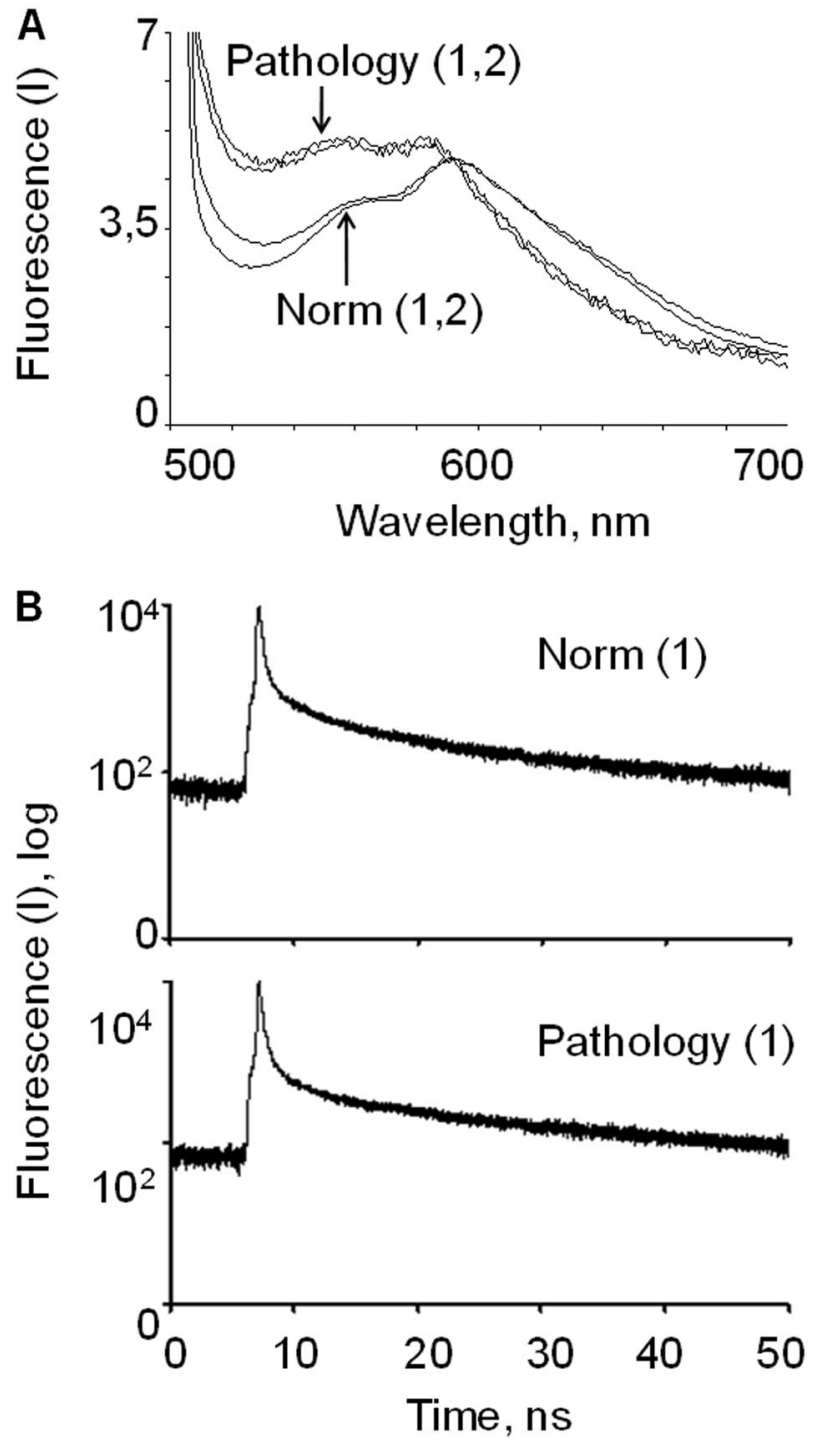

Fig. 4 a Fluorescence spectra and $\mathbf{b}$ fluorescence decay kinetic curves of LG fluorophores of RPE cells from human cadaver eyes. a Fluorescence was excited at $488 \mathrm{~nm}$, and the spectra were normalized to the fluorescence of $592 \mathrm{~nm}$. Two samples of RPE cell suspensions were from normal eyes (Norm) from a 74-year-old donor, and the other two were from eyes with AMD (Pathology) from a 75-year-old donor. b Fluorescence was excited at $485 \mathrm{~nm}$ (pulse duration, $30 \mathrm{ps}$ ), and kinetic curves were recorded at $540 \mathrm{~nm}$. One sample of RPE cell suspension was from a normal eye (Norm (1)), and the other was from an eye with AMD (Pathology (1))

studied two groups of LG fluorophores, belonging to nonoxidized and oxidized bisretinoids, which differ spectrally, in that the fluorescence spectrum maximum of oxidized bisretinoids is characterized by a significant shortwave shift [48]. The time parameters describe a group of compounds, which may have similar fluorescent properties and temporal fluorescence decay characteristics, rather than individual components of the system.

Figure $4 \mathrm{~b}$ shows examples of fluorescence decay kinetic curves of LG fluorophores in RPE cell suspensions from a 
Table 1 Fluorescence lifetime parameters (fluorescence excitation at $485 \mathrm{~nm}$ ) of LG fluorophores in suspensions of RPE cells from normal and AMD cadaver eyes

\begin{tabular}{lllllll}
\hline $\begin{array}{l}\text { RPE cell suspension } \\
\text { sample }\end{array}$ & $\tau_{1}, \mathrm{~ns}$ & $A_{1}, \%$ & $\tau_{2}, \mathrm{~ns}$ & $A_{2}, \%$ & $\tau_{3}, \mathrm{~ns}$ & $A_{3}, \%$ \\
\hline $\begin{array}{l}\text { Detection at 540 nm } \\
\quad\end{array}$ & & & & & & \\
$\quad$ Norm (1) & 0.7 & 0.6 & 5.1 & 19.4 & 10.1 & 80.0 \\
$\quad$ Norm (2) & 0.6 & 0.4 & 5.4 & 17.6 & 10.3 & 81.0 \\
$\quad$ Pathology (1) & 1.1 & 0.7 & 3.3 & 12.0 & 9.7 & 87.3 \\
$\quad$ Pathology (2) & 0.9 & 0.8 & 4.8 & 12.2 & 9.8 & 87.0 \\
Detection at 620 nm & & & & & & \\
$\quad$ Norm (1) & 0.8 & 7.0 & 3.0 & 57.0 & 8.7 & 36.0 \\
$\quad$ Norm (2) & 0.6 & 7.0 & 2.5 & 55.0 & 8.1 & 38.0 \\
$\quad$ Pathology (1) & 0.4 & 3.0 & 1.9 & 42.0 & 6.3 & 55.0 \\
$\quad$ Pathology (2) & 0.5 & 4.0 & 2.1 & 47.0 & 6.4 & 49.0 \\
\hline
\end{tabular}

normal and an AMD eyes following fluorescence excitation at $485 \mathrm{~nm}$. The time parameters $\left(\tau_{2}\right.$ and $\left.\tau_{3}\right)$, obtained by approximating the kinetic curves with a three-exponential model curve, are shown in Table 1 . Although the $\tau_{2}$ and $\tau_{3}$ values were close to those for normal and AMD eyes, respectively, the contributions of individual components $\left(A_{2}\right.$ and $A_{3}$ ) differed. For example, the contribution $A_{2}$ of the exponential component $\tau_{2}$ was about $6 \%$ lower for AMD than for normal eyes for detection at $540 \mathrm{~nm}$ and about $12 \%$ lower for detection at $620 \mathrm{~nm}$. At the same time, the contribution $A_{3}$ of the exponential component $\tau_{3}$ was about $7 \%$ higher for AMD than for normal eyes for detection at 540 $\mathrm{nm}$ and about $15 \%$ higher for detection at $620 \mathrm{~nm}$. We previously showed that, in the chloroform extract of LGs, bisretinoid photooxidation and photodegradation products had the highest fluorescence lifetime values $\left(\tau_{3}=7.2 \pm 0.3\right.$ ns) $[49,50]$. These findings suggest that, in evaluating RPE cell suspensions, the fluorophores with a fluorescence lifetime characterized by $\tau_{3}$ values are also bisretinoid photooxidation and photodegradation products.

These findings indicate that fluorophores with the highest fluorescence lifetime values $\left(\tau_{3}\right)$, as measured for normal eyes and those with AMD, are photooxidation and photodegradation products of bisretinoids. To date, however, the nature of the fluorophores with a fluorescence lifetime characterized by $\tau_{2}$ remains unknown.

Consequently, our findings suggest that the contents of bisretinoid photooxidation and photodegradation products in LGs are higher in AMD than in normal eyes, a difference manifested as changes in the characteristics of fluorescence spectra of LGs (Figs. 2a and 4a) and in the parameters of fluorescence decay kinetic curves (Fig. 4b). That is, fluorescence excitation at $488 \mathrm{~nm}$ of samples from eyes with AMD results in an increase in the fluorescence intensity of the band at $556 \mathrm{~nm}$, as well as an increase in the contribution of photooxidation and photodegradation products to total fluorescence.

\section{Discussion}

FAF imaging provides detailed insight into the health of the RPE. The incorporation of FAF into clinical practice will depend on the development of a quantitative approach.

This study presents results that may be useful in determining the quantitative spectral characteristics of FAF imaging. Our results indicate that the fluorescence of LGs can show the accumulation of photooxidation and photodegradation products of LG bisretinoids, and therefore may be an indicator of disease progression. Changes in the quantitative and qualitative composition of fluorophores and their spectral characteristics are determined only by the presence or absence of pathological changes in the RPE, but are independent of age. These patterns have characteristic and reproducible features that can be used as diagnostic indicators of visual pathology.

This study described two methods of identifying increased levels of bisretinoid photooxidation and photodegradation products in LGs from RPE cells in eyes with AMD. The first method was to compare the fluorescence intensity of the FAF, whereas the second method was to measure the lifetime of this fluorescence. Although both methods can form a basis for the development of the spectral analysis of the FAF, these methods differ in the complexity of analysis, the reproducibility of the data, and the uniqueness of interpretation of these data.

The fluorescence intensity method can make possible the spectral analysis of FAF, without regard to individual spectral components of LGs. The total fluorescence characteristics have reproducible features for normal eyes, regardless of age, and could be considered a diagnostic criterion.

The fluorescence lifetime method is less straightforward. In this method, complex experimental kinetic dependences that reflect a large number of fluorophores contained in LGs are approximated by relatively simple three-exponential curves. This method cannot, however, yield reliable quantitative diagnostic criteria. Despite this analysis also showing differences between normal and AMD eyes, this method is more complex and ambiguous.

\section{Summary}

\section{What was known before}

- Fundus autofluorescence imaging allows to identify early phenotypic changes in retinal pigment epithelium, occurring prior to the progression of the disease. 
- Analysis of fundus autofluorescence patterns can provide detailed qualitative information that allows the detection of areas of pathology, thereby differentiating among different types of ocular disease.

- At present, however, it is not possible to quantify the detected changes.

- The degree of disease progression must be assessed subjectively by an expert, who compares patterns with those of normal eyes.

\section{What this study adds}

- There is a possibility to improve the fundus autofluorescence technique to obtain additional information from the total fluorescence patterns.

- Quantitative determination of increases in photooxidation and photodegradation products of bisretinoids in lipofuscin granules may be used to establish quantitative diagnostic criteria for degenerative processes in the retina and retinal pigment epithelium even in the absence of visible signs of the disease.

Acknowledgements This work was supported by the Russian Foundation for Basic Research (No. 15-29-03831).

\section{Compliance with ethical standards}

Conflict of interest The authors declare that they have no conflict of interest.

\section{References}

1. Schmitz-Valckenberg S, Holz FG, Fitzke FW. Perspectives in imaging technologies. In: Holz FG, Schmitz-Valckenberg S, Spaide RF, Bird AC, editors. Atlas of fundus autofluorescence imaging. Berlin: Springer; 2007. p. 331-8.

2. Lois N, Holder GE, Bunce C, Fitzke FW, Bird AC. Phenotypic subtypes of Stargardt macular dystrophy-fundus flavimaculatus. Arch Ophthalmol. 2001;119:359-69.

3. Von Ruckmann A, Fitzke FW, Bird AC. In vivo fundus autofluorescence in macular dystrophies. Arch Ophthalmol. 1997;115:609-15.

4. Holz FG, Fleckenstein M, Schmitz-Valckenberg S, Bird AC. Evaluation of fundus autofluorescence images. In: Holz FG, Schmitz-Valckenberg S, Spaide RF, Bird AC, editors. Atlas of fundus autofluorescence imaging. Berlin: Springer; 2007. p. 71-76.

5. Kennedy CJ, Rakoczy PE, Constable IJ. Lipofuscin of the retinal pigment epithelium: a review. Eye. 1995;9:763-71.

6. Geng L, Wihlmark U, Algvere PV. Lipofuscin accumulation in iris pigment epithelial cells exposed to photoreceptor outer segments. Exp Eye Res. 1999;69:539-46.

7. König K. Clinical multiphoton tomography. J Biophot. 2008;1:13-23.

8. Koziol B, Markowicz M, Kruk J, Plytycz B. Riboflavin as a source of autofluorescence in Eisenia fetida coelomocytes. Photochem Photobiol. 2006;82:570-3.
9. Barker FM 2nd, Snodderly DM, Johnson EJ, Schalch W, Koepcke $\mathrm{W}$, Gerss J, et al. Nutritional manipulation of primate retinas, V: effects of lutein, zeaxanthin, and n-3 fatty acids on retinal sensitivity to blue-light-induced damage. Invest Ophthalmol Vis Sci. 2011;52:3934-42.

10. Tong Y, Ben Ami T, Hong S, Heintzmann R, Gerig G, Ablonczy $Z$, et al. Hyperspectral autofluorescence imaging of drusen and retinal pigment epithelium in donor eyes with age-related macular degeneration. Retina. 2016;36(Suppl. 1):S127-S136.

11. Bazan HEP, Bozan NG, Feeney-Burns L, Berman ER. Lipids in human lipofuscin-enriched subcellular fractions of two age populations. Comparison with rod outer segments and neural retina. Invest Ophthalmol Vis Sci. 1990;31:1433-43.

12. Eldred GE, Katz ML. Fluorophores of the human retinal pigment epithelium: separation and spectral characterization. Exp Eye Res. 1988;47:71-86.

13. Ng KP, Gugiu B, Renganathan K, Davies MW, Gu X, Crabb JS, et al. Retinal pigment epithelium lipofuscin proteomics. Mol Cell Proteom. 2008;7:1397-405.

14. Sparrow JR, Wu Y, Kim CY, Zhou J. Phospholipid meets all-trans retinal: the making of RPE bisretinoids. J Lipid Res. 2009;51:247-61.

15. Warburton S, Southwick K, Hardman RM, Secrest AM, Grow $\mathrm{RK}$, Xin $\mathrm{H}$, et al. Examining the proteins of functional retinal lipofuscin using proteomic analysis as a guide for understanding its origin. Mol Vis. 2005;11:1122-34.

16. Wu Y, Fishkin NE, Pande A, Pande J, Sparrow JR. Novel lipofuscin bisretinoids prominent in human retina and in a model of recessive Stargardt disease. J Biol Chem. 2009;284:20155-66.

17. Feldman TB, Yakovleva MA, Arbukhanova PM, Borzenok SA, Kononikhin AS, Popov IA, et al. Changes in spectral properties and composition of lipofuscin fluorophores from human retinal pigment epithelium with age and pathology. Anal Bioanal Chem. 2015;407:1075-88.

18. Lamb LE, Simon JD. A2E: a component of ocular lipofuscin. Photochem Photobiol. 2004;79:127-36.

19. Sakai N, Decatur J, Nakanishi K, Eldred GE. Ocular age pigment "A2E": an unprecedented pyridinium bisretinoid. J Am Chem Soc. 1996;118:1559-60.

20. Sparrow JR, Kim SR, Cuervo AM, Bandhyopadhyayand U. A2E, a pigment of RPE lipofuscin, is generated from the precursor, A2PE by a lysosomal enzyme activity. Adv Exp Med Biol. 2008;613:393-8.

21. Avalle LB, Wang Z, Dillon JP, Gaillard ER. Observation of A2E oxidation products in human retinal lipofuscin. Exp Eye Res. 2004;78:895-8.

22. Dillon JP, Wang Z, Avalle LB, Gaillard ER. The photochemical oxidation of A2E results in the formation of a 5,8,5', $8^{\prime}$,-bis-furanoid oxide. Exp Eye Res. 2004;79:537-42.

23. Yoon KD, Yamamoto K, Ueda K, Zhou J, Sparrow JR. A novel source of methylglyoxal and glyoxal in retina: implications for age related macular degeneration. PLoS ONE. 2012;7:e41309.

24. Wang Z, Keller LMM, Dillon J, Gaillard ER. Oxidation of A2E results in the formation of highly reactive aldehydes and ketones. Photochem Photobiol. 2006;82:1251-7.

25. Wu Y, Yanase E, Feng X, Siegel MM, Sparrow JR. Structural characterization of bisretinoid $\mathrm{A} 2 \mathrm{E}$ photocleavage products and implications for age-related macular degeneration. Proc Natl Acad Sci USA. 2010;107:7275-80.

26. Kim SR, Jang YP, Jockusch S, Fishkin NE, Turro NJ, Sparrow JR. The all-trans-retinal dimer series of lipofuscin pigments in retinal pigment epithelial cells in a recessive Stargardt disease model. Proc Natl Acad Sci USA. 2007;104:19273-8.

27. Sparrow JR, Wu Y, Nagasaki T, Yoon KD, Yamamoto K, Zhou J. Fundus autofluorescence and the bisretinoids of retina. Photochem Photobiol Sci. 2010;9:1480-9. 
28. Yamamoto K, Yoon KD, Ueda K, Hashimoto M, Sparrow JR. A novel bisretinoid of retina is an adduct on glycerophosphoethanolamine. Invest Ophthalmol Vis Sci. 2011;52:9084-90.

29. Holz FG, Schmitz-Valckenberg S, Spaide RF, Bird AC. Atlas of fundus autofluorescence imaging. Berlin, Springer, 2007.

30. Mühlhoff D. Laser scanning ophthalmoscope. US patent 6,337,920 B1, 2002.

31. Park SP, Siringo FS, Pensec N, Hong IH, Sparrow J, Barile G, et al. Comparison of fundus autofluorescence between fundus camera and confocal scanning laser ophthalmoscope-based systems. Ophthalmic Surg Lasers Imaging Retin. 2013;44:536-43.

32. Marmorstein AD. Apparatus and method for ratiometric quantitation of elicited autofluorescence of the eye. US patent 2003/ 0004418 A1, 2003.

33. Schweitzer D, Kolb A, Hammer M, Thamm E. Method and apparatus for determining fluorophores on objects, especially on the living ocular fundus. US Patent 6,371,615 B1, 2002.

34. Sharifzadeh M, Gellermann W. Systems and methods for optical detection of lipofuscin concentration in a subject's eye. US patent 7,914,147 B2, 2011.

35. Zuckerman R. Apparatus for the non-invasive measurement of tissue function and metabolism by determination of steady-state fluorescence anisotropy. US patent 8,309,346 B2, 2012.

36. Marmorstein AD, Marmorstein LY, Sakaguchi H, Hollyfield JG. Spectral profiling of autofluorescence associated with lipofuscin, bruch's membrane, and sub-RPE deposits in normal and AMD eyes. Invest Ophthalmol Vis Sci. 2002;43:2435-41.

37. Dysli C, Quellec G, Abegg M, Menke MN, Wolf-Schnurrbusch U, Kowal J, et al. Quantitative analysis of fluoresceence lifetime measurements of the macula using the fluorescence lifetime imaging ophthalmoscope in healthy subjects. Invest Ophthalmol Vis Sci. 2014;55:2106-13.

38. Dysli C, Wolf S, Berezin MY, Sauer L, Hammer M, Zinkernagel MS. Fluorescence lifetime imaging ophthalmoscopy. Prog Retin Eye Res. 2017;58:4856-62.

39. Schweitzer D, Schenke S, Hammer M, Schweitzer F, Jentsch S, Birckner E, et al. Towards metabolic mapping of the human retina. Microsc Res Tech. 2007;70:410-9.

40. Schweitzer D, Gaillard ER, Dillon J, Mullins RF, Russell S, Hoffmann B, et al. Time-resolved autofluorescence imaging of human donor retina tissue from donors with significant extramacular drusen. Invest Ophth Vis Sci. 2012;53:3376-86.
41. Schweitzer D, Quick S, Schenke S, Klemm M, Gehlert S, Hammer M, et al. Comparison of parameters of time-resolved autofluorescence between healthy subjects and patients suffering from early AMD. Ophthalmology. 2009;106:714-22.

42. Schweitzer D, Deutsch L, Klemm M, Jentsch S, Hammer M, Peters S, et al. Fluorescence lifetime imaging ophthalmoscopy in type 2 diabetic patients who have no signs of diabetic retinopathy. J Biomed Opt. 2015;20:61106.

43. Ramm L, Jentsch S, Augsten R, Hammer M. Fluorescence lifetime imaging ophthalmoscopy in glaucoma. Graef Arch Clin Exp. 2014;252:2025-6.

44. Jentsch S, Schweitzer D, Schmidtke KU, Peters S, Dawczynski J, Bar KJ, et al. Retinal fluorescence lifetime imaging ophthalmoscopy measures depend on the severity of Alzheimer's disease. Acta Ophthalmol (Copenh). 2014;93:241-7.

45. Russian Federation Law N 4180-I dated 22.12.1992, "On human organs or tissue transplantation" (with modifications and additions). http://base.garant.ru/136366/.

46. Folch J, Lees M, Stanley GHS. A simple method for the isolation and purification of total lipids from animal tissues. J Biol Chem. 1957;226:497-509.

47. Parish CA, Hashimoto M, Nakanishi K, Dillon J, Sparrow JR. Isolation and one-step preparation of $\mathrm{A} 2 \mathrm{E}$ and iso-A2E, fluorophores from human retinal pigment epithelium. Proc Natl Acad Sci USA. 1998;95:14609-13.

48. Feldman TB, Yakovleva MA, Dontsov AE, Ostrovsky MA. Fluorescence emission and excitation spectra of fluorophores of lipofuscin granules isolated from retinal pigment epithelium of human cadaver eyes. Russ Chem Bull Int Ed. 2010;59: 276-83.

49. Yakovleva MA, Feldman TB, Arbukhanova PM, Borzenok SA, Kuzmin VA, Ostrovsky MA. Estimation of fluorescence lifetime of lipofuscin fluorophores contained in lipofuscin granules of retinal pigment epithelium of human cadaver eyes without signs of pathology. Dokl Biochem Biophys. 2017;472:19-22.

50. Yakovleva MA, Feldman TB, Arbukhanova PM, Borzenok SA, Kuzmin VA, Ostrovsky MA. The fluorescence lifetime of lipofuscin granule fluorophores contained in the retinal pigment epithelium cells from human cadaver eyes in normal state and in the case of visualized pathology. Dokl Biochem Biophys. 2017;474:239-43. 